\title{
Hypervitaminosis A Causes Reversible Changes in the Density of Hepatocytes and Hepatic Stellate Cells of Albino Rats (Rattus norvegicus)
}

\author{
Jeremiah K. Munguti, ${ }^{1}$ Moses M. Obimbo, ${ }^{1}$ Paul O. Odula, ${ }^{1}$ Andrew N. Makanya, ${ }^{2}$ William O. Sibuor ${ }^{3}$ \\ 1Department of Human Anatomy, University of Nairobi, Nairobi, Kenya \\ 2Department of Veterinary Anatomy and Physiology, University of Nairobi, Nairobi, Kenya \\ ${ }^{3}$ School of Medicine, University of Nairobi, Nairobi, Kenya
}

Disclose and conflicts of interest: none to be declared by all authors

\begin{abstract}
Background: hypervitaminosis A causes hyperplasia and vacuolation of hepatocytes and hepatic stellate cells (HSCs). The temporal sequence of these changes and the sequalae of their spontaneous reversion, however, remain largely unquantified. Methods: ethical approval was obtained from the Biosafety, Animal Use and Ethics Committee of the School of Veterinary Medicine at the University of Nairobi. A total of 45 adult albino rats were randomly divided into 3 groups. In groups $A$ and B, 300,000 IU $/ \mathrm{Kg}$ of vitamin A were administered to the rats every other day via subcutaneous injection for 4 and 8 weeks respectively. The rats in group $C$ formed the control group. In total, 5 rats from group A were euthanized weekly at weeks 2, 4, 6 and 8, while those from group B were euthanized at weeks 6, 8, 10 and 12. Multistage systematic uniform random sampling was used to select harvested liver segments that were processed for histological staining. A one-way analysis of variance (ANOVA) was used to compare cell density in the 3 groups and over time. The Tukey test was used to detect the differences between the groups. Values of $p<0.05$ were considered statistically significant at $95 \%$ confidence interval ( $95 \% \mathrm{Cl}$ ). Results: in both the acutely- and chronically-exposed groups, there was a significant increaseinhepatocyte $(p<0.001)$ and HSC $(p-0.066)$ density over time. Stopping the exposure resulted in a significant decline in both cell lines $(p<0.05)$. Compensatory hyperplasia and hypertrophy of these cells emanated from the periportal areas.

Conclusion: hepatocytes are more adversely affected by hypervitaminosis A compared to HSC, which reflects their active role in the metabolism of vitamin A.
\end{abstract}

Keywords: Hepatocyte; Stellate cells; Vitamin A.

\section{Introduction}

Vitamin A is a fat-soluble vitamin essential for vision, growth, cellular differentiation and the integrity of the immune system. ${ }^{1}$ Its established tolerable upper limit (UL) of safety is of $\sim 2,800$ to 3,000 $\mu \mathrm{g} /$ day for adult men and women. ${ }^{2}$ The prevalence of exceeding this limit in populations with adequate dietary content of vitamin A has been shown to range between $10 \%$ and $31 \%$. $^{3,4}$ Furthermore, because of its ability to act as an antioxidant, vitamin A derivatives have been used in the prophylaxis and treatment of photoaging, certain skin cancers and other skin disorders. ${ }^{5}$

Following such therapeutic uses, several cases of vitamin-A- induced hepatotoxicity have been reported in the absence of other clinical signs and symptoms. ${ }^{6}$ Overt liver cirrhosis has also been documented following prolonged intake of high doses vitamin A supplements. ${ }^{7,8}$

The hepatotoxicity profile includes hyperplasia of the hepatocytes and hepatic stellate cells (HSCs), ${ }^{1,9}$ and an increase in the number and size of the vacuoles found in these cells.10 The temporal sequence of these changes in response to hypervita-minosis A, however, is yet to be quantified. Furthermore, the sequalae of the spontaneous reversion of these cellular changes following cessation of exposure to hypervitaminosis A remains largely unknown. The present study, therefore, aimed at determining the temporal changes in the density of hepatocytes and HSCs and their vacuolation following exposure to high doses of vitamin A.

\section{Materials and Methods}

A total of 45 adult male rats were used in the present study. Ethical approval to conduct the study was obtained from the Biosafety, Animal Use and Ethics Committee of the School of Veterinary Medicine at the University of Nairobi. Only rats that met the inclusion criteria were recruited into the experiments.

The selected rats were randomly divided into 3 groups: A (20 rats), B (20 rats), and C (5 rats). The rats in group $\mathrm{A}$ represented the acutely-exposed animals, and high dose vitamin A (300,000 IU/ $\mathrm{Kg})^{8}$ were administered to them every other day via subcutaneous injection for 4 weeks, with half of them being followed up for a further 4 weeks without treatment. The rats in group $B$, which represented 
persistent hypervitaminosis $\mathrm{A}$, were also submitted to high doses vitamin A $(300,000 \mathrm{IU} / \mathrm{Kg})^{8}$ every other day via subcutaneous injection for 8 weeks. Half of the members of this group were then followed up for 4 additional weeks without treatment. Group $\mathrm{C}$ was the control group, which was submitted to subcutaneous injection of sterile normal saline every other day over the duration of the study. All rats were maintained on a normal diet and fed ad libitum. Two rats from the control group were used for the baseline results.

Every week, 5 rats from group A were euthanized using gaseous halothane (1-3\%) at weeks 2, 4, 6 and 8, while those fromgroup B were euthanized at weeks 6 , 8,10 and 12 . The liver was then harvested en masse and stored in normal saline overnight. The preparation and selection of the liver segments (5 segments per liver) was performed using multistage system-atic uniform random(SUR) sampling, as previously described..$^{11}$ The selected segments were then prepared for staining using hematoxylin and eosin.

Photomicrographs of the stained sections were taken using a 5-megapixel Canon (Ōta, Tokyo, Japan) digital camera mounted on a photomicroscope. The calibration was set at $150 \mu \mathrm{m}$ for images taken at a magnification of 400X. In total, six images per section were selected using the SUR sampling method - three within the vicinity of a central vein and three within the vicinity of a portal vein.

The estimation of the density of the hepatocytes and HSCs was performed using the Cavalieri's principle of point counting. ${ }^{9,10}$ We used the ImageJ software (public domain) to analyze the selected histological area by using a superimposed grid on the digital image on a monitor screen as previously described ${ }^{11}$ (Fig. 1). The superimposed image on the grid yielded 15 unit areas. Each unit area on the grid was of $285,967.77 \mu \mathrm{m}^{2}$. The second unit areas for every section were selected to count the cells and, after that, every third one, until a total of 5 unit areas per slide were studied. The average cellular count $/ \mu \mathrm{m}^{2}$ of the 5 unit areas was then used as the cell density of that image. The area densities of the cells were evaluated by two researchers who were unaware of the source of the tissue samples.

\section{Statistical Analysis}

Data obtained from the stereological methods previously described were inserted into the Statistical Package for the Social Sciences (SPSS, IBM Corp., Armonk, NY, US) software, version 21.0, for coding, tabulation and statistical analysis. The measurements were expressed in numbers/mm2.The normality of the data was determined using histograms and box plots, after which means and the standard deviations were then determined. The one-way analysis of variance (ANOVA) was used to compare the density of the hepatocytes and HSCs for the 3 groups, and the changes in the density over time. When a significant difference was shown with the ANOVA, the Tukey test was used as a posthoc test to detect the groups that presented a significant difference between each other. Values of $p<0.05$ were considered significant at a $95 \%$ confidence interval $(95 \% \mathrm{CI})$. The results were presented in micrographs, tables and graphs.

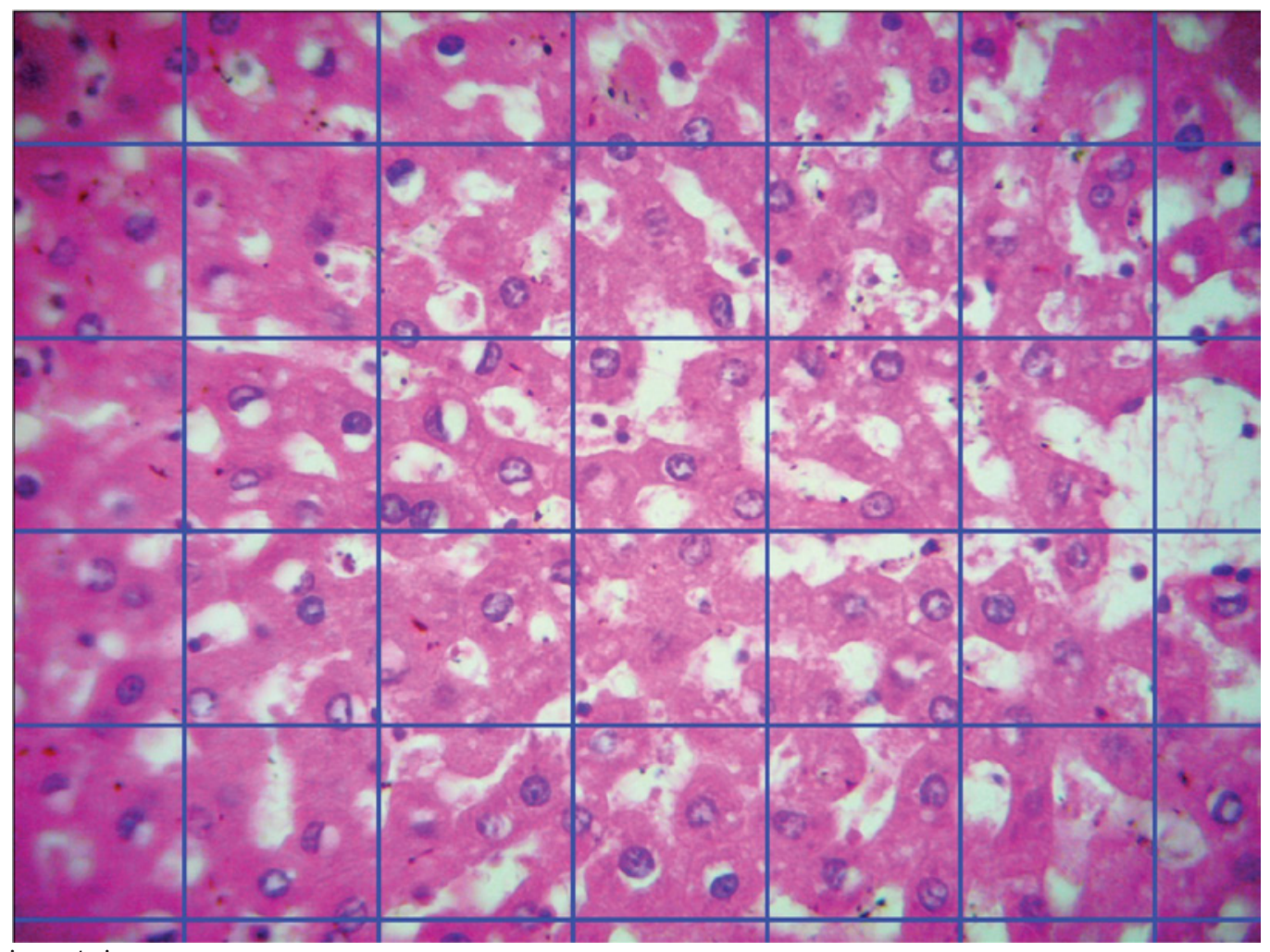

Figure 1. Grid counting technique. 


\section{Results}

The density of the hepatocytes and HSCs for the control group remained relatively constant over the entire study period (Figure 2A, B). The hepatocytes were heterogeneously arranged in cords separated by sinusoids. The HSCs were located in the perisinusoidal space, and appeared deeply eosinophilic. There was no notable difference in their distribution between the periportal and pericentral areas.

Acute exposure to high doses of vitamin A resulted in an increase in the hepatocyte density $(p<0.001)$ (Figure 4). The hepatocytes closer to the central veins were more vacuolated than those in the periportal areas (Fig. 2C, D). Conversely, the hepatocytes near the periportal areas were more densely packed andwere of varied sizes. The hepatocytes further showed a temporal increase in their binucleation. Adjacent biliary epithelial cells appeared hypertrophic relative to the controls. Discontinuing the acute exposure resulted in a decline of the hepatocyte density both at week $6(p=0.205)$ and at week $8(p=0.901)$ (Figure
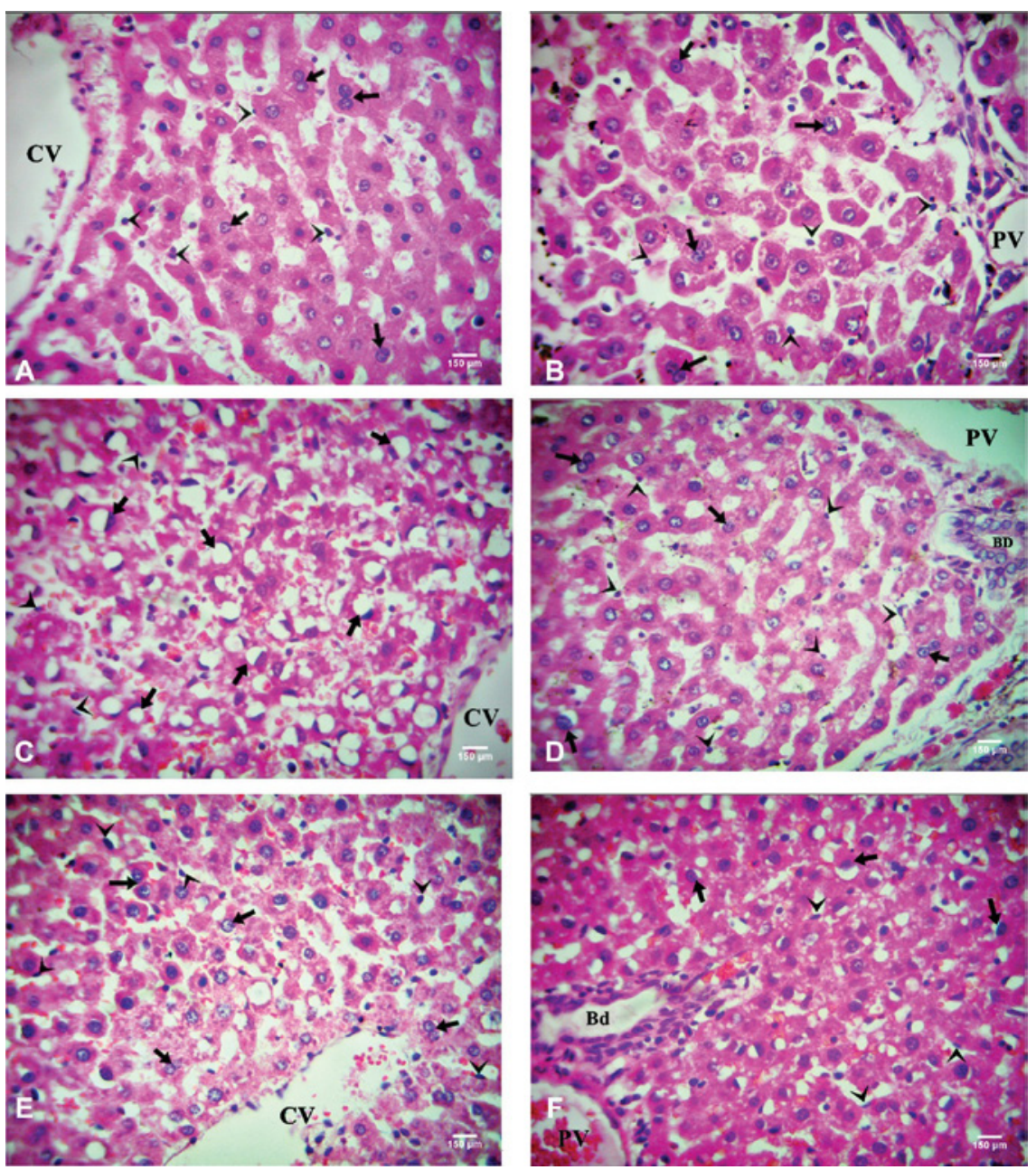

Figure 2. Hepatocyte and HSC cellular changes following acute exposure and reversal. The hepatocytes (arrows) in the control rats were heterogeneously distributed between the central veins (CVs) (A)andportal veins (PVs) (B), and were arranged in cords, while the hepatic stellate cells (HSCs; arrow heads) were located in the perisinusoidal spaces. Acute exposure to hypervitaminosis A (4 weeks) led to increased vacuolation of the hepatocytes and HSCS closest to the CVs (C), while cells around the pericentral areas (D) were more densely packed and presented lower vacuolation. Discontinuing the administration of excess vitamin A resulted in a reversal of the aforementioned trend, with restoration of the hepatic cords. The cells were, however, more vacuolated around the CVs ( $E$ ) than around a PV ( $F$ ) (hematoxylin and eosin [H\&E] stain). Scale: $150 \mu \mathrm{m}$. (A and B - control rats; C and D - at the end of 4 weeks of injection of high doses of vitamin A; E and F - 4 weeks after the discontinuation of the acute exposure to high doses of vitamin A). 



Figure 3. Cellular changes following persistent exposure and reversal. Persistent exposure to hypervitaminosis $A$ (8 weeks) resulted in further vacuolation of the hepatocytes (arrows) and HSCs (arrow heads) around the CVs (A, B) that extended to the PVs. Rebound hyperplasia and cellular necrosis occurred in tandem as evidenced by the closely-packed cells around the PV and the loss of the sharp border demarcating the hepatocytes from the surrounding stroma respectively. The HSCS were more uniformly distributed across the pericentral and periportal areas, and took the shape of a signet ring. There was also massive distortion of the hepatic cords and apparent loss of sinusoidal space (H\&E stain). Scale: $150 \mu \mathrm{m}$. Discontinuing the exposure to high doses of vitamin A resulted in restoration of the hepatic cords 4 weeks later (C, D). However, the hepatocytes and HSCs around the CVs were more vacuolated, while restorative hyperplasia of the said cells seemed to emanate from around the PV (H\&E stain). Scale: $150 \mu \mathrm{m}$. (A and B - after 8 weeks of exposure to hypervitaminosis A; C and D - recovery 4 weeks after 8 weeks of exposure to hypervitaminosis A).



Control $\longrightarrow$ Acute Intervention/Reversal $\longrightarrow$ Sustained Intervention/Reversal

Figure 4. General trend of the hepatocyte density over time.

The HSC density also showed a time-dependent increase that reversed considerably with the discontinuation of the administration of the vitamin (Figure 3C). The increase in the HSC density was more marked in the periportal zones compared with the pericentral ones. These cells became increasingly vacuolated with time, with accompanying displacement of the nucleus to the periphery, and they took the shape of a signet ring by week 6 . The hyperplasia of the HSC seemed to occur in clusters. Persistent exposure to high doses of vitamin A resulted in a minimal decline of the HSC density at week 6, which then slightly rebounded at week $8(p>0.05)$ (Figure 5). Discontinuing the administration of high doses of vitamin A after week 8 , however, resulted in a significant decline in the HSC density by week $12(p-0.013)$ (Figure 3C), which was accompanied by a considerable reduction in their vacuolation (Figs. 3C \& D).

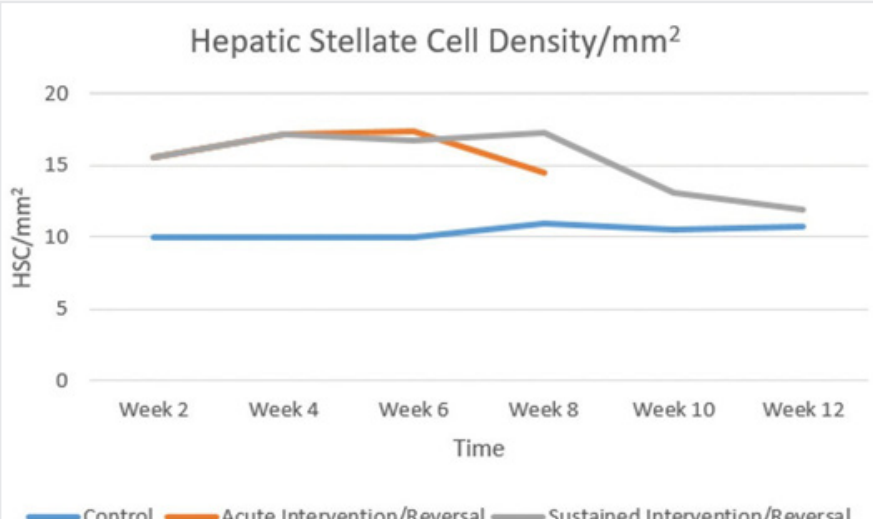

Figure 5. General trend of the hepatic stellate density over time. 


\section{Discussion}

Hepatic toxicity secondary to retinoic acid has been reported to range from a simple elevation of hepatic enzymes to liver cirrhosis requiring liver transplant., ${ }^{2,4,12}$ The risk factors to retinoic acid poisoning include chronic renal failure, enhanced dietary intake and use of retinoic acid derivatives in the management of certain dermatologic ailments. ${ }^{3,5,7}$ The reversible cellular hyperplasia with hypervitaminosis A as seen in our findings reflects the liver's remarkable ability to regenerate itself following injury. These findings are in keeping with previous reports documenting the liver's regenerative capacity following partial hepatectomy and administration of hepatotoxic chemicals. ${ }^{13,14}$ However, the current findings further showed a biphasic rebound hyperplasia with persistent exposure to hypervitaminosis A, with the liver being able to recover from the decline in cellular numbers at week 6 of exposure. This observation highlights the liver's remarkable regenerative capacity in the face of persistent chemotoxicity. Hepatocyte hyperplasia was additionally accompanied by hypertrophy of both the hepatocytes and biliary duct cells, events that have been reported to be vital for liver regeneration, as these mature cell lines can function as facultative stem cells for each other.13,15 Hepatocyte hypertrophy was also associated with increased vacuolation, which has previously been described as an adaptation of these cells to resist further damage. ${ }^{16,17}$ Such hypertrophy resulted in an apparent reduction in hepatocyte density, which might explain the decline in total hepatocyte numbers that was most prominent by week 6 of exposure.

The increased cellularity of the periportal areas accompanied by more multinucleated hepatocytes following exposure to hypervitaminosis A is in line with previous findings that reported that liver regeneration occurs via 'waves of mitosis' beginning in the periportal area, which progress toward the pericentral area. ${ }^{18,19}$ Moreover, more recent research ${ }^{20}$ has further revealed that increasingly less hepatocytes are recruited in subsequent 'waves' until the hepatic mass is restored. This, however, largely depends on whether the hepatic trauma occurs as a continuum. ${ }^{2} 0$ Continued hepatocyte proliferation in the face of persistent exposure to hypervitaminosis A in the current study may explain their biphasic increase as seen at weeks 4 and 8. An overshoot of this proliferative response is corrected by inducing hepatocyte apoptosis, ${ }^{21}$ which might explain the presence of apoptotic cells interspersed in the periportal areas and the eventual decline of hepatocyte density with the end of the exposure to hypervitaminosis A.

The rapid rise in HSC density by week two of exposure to high doses of vitamin A reflects the vital role of these cells in the storage of vitamins. ${ }^{7}$ The findings of the current study quantified the previously reported qualitative time and dosedependent hyperplasia of these cells following exposure to hypervitaminosis A in rodents. ${ }^{10,22}$ The HSC hyperplasia observed in the present study occurred more in the periportal zone in acute states, but was more diffuse with persistent exposure. This differs from the effects in humans in one study, ${ }^{22}$ in which an intake of excess vitamin A resulted in lobular hyperplasia, while chronic exposure led to localized hyperplasia limited mainly to the portal spaces and/or within the fibrous septa. The hyperplasia of these cells in hypervitaminosis A has been known to occur even in extrahepatic tissues, further emphasizing the significance of the HSCs in the metabolism of vitamin $\mathrm{A}^{10}$

Discontinuing exposure to vitamin A in both the acutely-and chronically-exposed animals resulted in a decline in HSC numbers albeit not immediately. This is attributable to the body's inability to effectively dispose of excess retinol, ${ }^{23}$ and might explain the continued clinical picture of retinoic acid poisoning long after the discontinuation of the exposure. ${ }^{24}$ Apoptosis of the HSC has ,however, been credited with the eventual decline of the activated HSCs during resolution from hepatic injury. ${ }^{24,25}$ This is mediated partly by the resolution of the inflammatory response and the recovery of hepatocytes, which are the key sources of cytokines responsible for the activation of HSCs.

Similar to the hepatocytes and as previously reported,$^{10}$ the HSCs responded to acute states of hypervitaminosis A by having a reversible increase in the number and size of their cytoplasmic vacuoles. Such vacuolation is routinely accompanied by inflammatory cellular infiltration and pericellular collagen fiber deposition, ${ }^{26}$ and has previously been associated with cases of hepatic fibrosis, portal hypertension and liver cirrhosis. ${ }^{7,8,27,28}$ We also observed that there was markedly reduced vacuolation of the HSCs by week 8 of exposure, possibly signifying the duration over which these cells potentially converted into myofibroblasts in the current study. This conversion, however, is known to not only be time-dependent but also dosedependent; 22 hence, our findings may be inconclusive to establish the time it takes for the transformation of the HSCs into myofibroblasts occurs.

\section{Conclusion}

Hepatocytes are more adversely affected by hypervitaminosis A compared to HSCs, which reflects their active role in the metabolism of vitamin A. This may equally affect the liver's metabolic capacity, further affecting its ability to handle the circulating vitamin A. Future studies should, therefore, incorporate the measurement of hepatic enzymes when monitoring the progress of vitamin A toxicity. 


\section{References}

1. Raoofi A, Asadi F, Mardjanmehr SH, Kazempoor R. The effects of hypervitaminosis $A$ in sheep following intramuscular administrations of vitamin A. Food Chem Toxicol 2010;48(01):193-195.

2. Institute of Medicine (US) Panel on Micronutrients. Dietary Reference Intakes for Vitamin A, Vitamin K, Arsenic, Boron, Chromium, Copper, Iodine, Iron, Manganese, Molybdenum, Nickel, Silicon, Vanadium, and Zinc. Washington (DC): National Academies Press (US); 2001.

3. Ahluwalia N, Herrick KA, Rossen LM, et al. Usual nutrient intakes of US infants and toddlers generally meet or exceed Dietary Reference Intakes: findings from NHANES 2009-2012. Am J Clin Nutr 2016;104(04):1167-1174.

4. Cheruvattath R, Orrego M, Gautam M, et al. Vitamin A toxicity: when one a day doesn't keep the doctor away. Liver Transpl 2006; 12(12):1888-1891.

5. Murphy SP, White KK, Park S-Y, Sharma S. Multivitamin-multimineral supplements' effect on total nutrient intake. Am J Clin Nutr 2007;85(01):280S-284S.

6. Keller KL, Fenske NA. Uses of vitamins A, C, and E and related compounds in dermatology: a review. J Am Acad Dermatol 1998; 39(4 Pt 1):611-625.

7. Levine PH, Delgado Y, Theise ND, West AB. Stellate-cell lipidosis in liver biopsy specimens. Recognition and significance. Am J Clin Pathol 2003;119(02):254-258.

8. Nagy NE, Holven KB, Roos $N$, et al. Storage of vitamin $A$ in extrahepatic stellate cells in normal rats. J Lipid Res 1997;38 (04):645658.

9. Sheth A, Khurana R, Khurana V. Potential liver damage associated with over-the-counter vitamin supplements. J Am Diet Assoc 2008;108(09):1536-1537.

10. Gundersen HJG, Bagger P, Bendtsen TF, et al. The new stereological tools: disector, fractionator, nucleator and point sampled intercepts and their use in pathological research and diagnosis. APMIS 1988;96(10):857-881.

11. Friedman SL. Evolving challenges in hepatic fibrosis. Nat Rev Gastroenterol Hepatol 2010;7(08):425-436.

12. Castaño G, Etchart C, Sookoian S. Vitamin A toxicity in a physical culturist patient: a case report and review of the literature. Ann Hepatol 2006;5(04):293-395.

13. Geubel AP, De Galocsy C, Alves N, Rahier J, Dive C. Liver damage caused by therapeutic vitamin A administration: estimate of dose related toxicity in 41 cases. Gastroenterology 1991;100 (06):1701-1709. 14. Graham-Maar RC, Schall JI, Stettler N, Zemel BS, Stallings VA. Elevated vitamin $A$ intake and serum retinol in preadolescent children with cystic fibrosis. Am J Clin Nutr 2006;84(01): 174-182.
15. Grisham JW. A morphologic study of deoxyribonucleic acid synthesis and cell proliferation in regenerating rat liver; autoradiography with thymidine-H3. Cancer Res 1962; 22:842-849.

16. Higashi $N$, Imai $K$, Sato $M$, et al. Intralobular Distribution of Vitamin A-Storing Lipid Droplets in Hepatic Stellate Cells with Special Reference to Polar Bear and Arctic Fox. Comp Hepatol 2004;3(Suppl 1):S16.

17. Mandarim-de-Lacerda CA. Stereological tools inbiomedical research. An Acad Bras Cienc 2003;75(04):469-486

18. Michalopoulos GK. Liver regeneration. J Cell Physiol 2007;213 (02):286-300.

19. Miyaoka Y, Ebato K, Kato H, Arakawa S, Shimizu S, Miyajima A. Hypertrophy and unconventional cell division of hepatocytes underlie liver regeneration. Curr Biol 2012;22(13):1166-1175

20. Miyaoka Y, Miyajima A. To divide or not to divide: revisiting liver regeneration. Cell Div 2013;8(01):8.

21. Nayak NC, Sathar SA, Mughal S, Duttagupta S, Mathur M, Chopra $P$. The nature and significance of liver cell vacuolation following hepatocellular injury-an analysis based on observations on rats rendered tolerant to hepatotoxic damage. Virchows Arch 1996; 428(06):353-365.

22. Rabes HM. Kinetics of hepatocellular proliferation as a function of the microvascular structure and functional state of the liver. Ciba Found Symp 1977; •••(55):31-53

23.Sakamoto T, Liu Z, Murase N, et al. Mitosis and apoptosis in the liver of interleukin-6-deficient mice after partial hepatectomy. Hepatology 1999;29(02):403-411.

24. Tarlow BD, Pelz C, Naugler WE, et al. Bipotential adult liver progenitors are derived from chronically injured mature hepatocytes. Cell Stem Cell 2014;15(05):605-618.

25. Wright MC, Issa R, Smart DE, et al. Gliotoxin stimulates the apoptosis of human and rat hepatic stellate cells and enhances the resolution of liver fibrosis in rats. Gastroenterology 2001;121 (03):685698.

26. Hassan NF, Soliman GM, Okasha EF, Shalaby AM. Histological, immunohistiochemical and biochemical study of experimentally induced fatty liver in adult male albino rat and the possible protective role of pomegranate. J Microsc Ultrastruct 2018;6 (01):44-55.

27. Burillo Lorente J, Castellote Alonso J, Massanes Bohigas PR. [Portal hypertension and hepatic fibrosis caused by vitamin A]. Rev Clin Esp 1991;189(08):396-397.

28. García-Muñoz P, Bernal-Bellido C, Marchal-Santiago A, et al. Liver cirrhosis from chronic hypervitaminosis A resulting in liver transplantation: A case report. Transplant Proc 2019;51(01): 90-91.
Received: December 21, 2018

Accepted: September 4, 2019
Corresponding author

Jeremiah K. Munguti

Email: donaldjrmh86@gmail.com 